\title{
(2-Imidazolin-4-yl)phosphonates: Green Chemistry and Biology Walk Together ${ }^{\dagger}$
}

\author{
Andrea Bagán 1,*, Sònia Abás 1, Sergio Rodríguez-Arévalo 1, Gemma Rodríguez-Arévalo ${ }^{1}$, \\ Fotini Vasilopoulou 2, Christian Griñán-Ferré ${ }^{2}$, Mercè Pallàs ${ }^{2}$, Pilar Pérez-Lozano ${ }^{3}$, \\ Milica Radan ${ }^{4}$, Teodora Djikic ${ }^{4}$, Katarina Nikolić ${ }^{4}$ and Carmen Escolano ${ }^{1}$ \\ 1 Laboratory of Medicinal Chemistry (Associated Unit to CSIC), Department of Pharmacology, \\ Toxicology and Therapeutic Chemistry, Faculty of Pharmacy and Food Sciences, \\ and Institute of Biomedicine of the University of Barcelona (IBUB), 08028 Barcelona, Spain; \\ soniaabas88@gmail.com (S.A.); rodriguez.arevalo@ub.edu (S.R.-A.); gemma2_7@hotmail.com (G.R.-A.); \\ cescolano@ub.edu (C.E.) \\ 2 Pharmacology Section, Department of Pharmacology, Toxicology and Therapeutic Chemistry, \\ Faculty of Pharmacy and Food Sciences, and Institut de Neurociències, University of Barcelona, \\ 08028 Barcelona, Spain; ftn.vasilopoulou@gmail.com (F.V.); christian.grinyan@hotmail.com (C.G.-F.); \\ pallas@ub.edu (M.P.) \\ 3 Unit of Pharmaceutical Technology, Pharmacy and Pharmaceutical Technology Department, Faculty of \\ Pharmacy and Food Sciences, University of Barcelona, 08028 Barcelona, Spain; perezlo@ub.edu \\ 4 Department of Pharmaceutical Chemistry, Faculty of Pharmacy, University of Belgrade, \\ Vojvode Stepe 450, 11000 Belgrade, Serbia; milica.radan@pharmacy.bg.ac.rs (M.R.); \\ Teodora.djikic@pharmacy.bg.ac.rs (T.D.); katarina.nikolic@pharmacy.bg.ac.rs (K.N.) \\ * Correspondence: andreabaganp@gmail.com; Tel.: +34627208929 \\ + Presented at the 2nd Molecules Medicinal Chemistry Symposium (MMCS): Facing Novel Challenges in \\ Drug Discovery, Barcelona, Spain, 15-17 May 2019.
}

Published: 10 September 2019

2-Imidazoline-containing compounds constitute a valuable class of agents that modulate $\alpha_{2-}$ adrenergic receptors and often show a high affinity for imidazoline $\mathrm{I}_{2}$-receptors ( $\left.\mathrm{I}_{2}-\mathrm{IR}\right)$. Moreover, 2imidazolines are an important class of heterocyclic scaffolds found in natural product chemistry, coordination chemistry, and homogeneous catalysis. To meet the demand for 2-imidazolinecontaining compounds, different synthetic approximations were developed. In this work, we describe an efficient and user-friendly synthetic process involving the combination of isocyanidebased multicomponent reaction and microwave heating without the need of anhydrous atmosphere or additional solvents that generates unprecedented (2-imidazolin-4-yl)phosphonates [1].

We assessed the pharmacological profile and selectivity of the prepared compounds upon I2-IR. Owing to the outstanding high $\mathrm{I}_{2}$-IR affinity of one of the prepared compounds and high selectivity devoid to the $\alpha_{2}$-adrenoceptor of other compounds, markedly better than any described I2-IR ligand to date, (2-imidazolin-4-yl)phosphonates might be considered as a suitable scaffold for designing novel I2-IR ligands [2]. In addition, we demonstrated the effectiveness of two of the new $\mathrm{I}_{2}$-IR ligands in an in vivo female model for cognitive decline (SAMP8), and we analyzed the pathological biomarkers for neurodegeneration. This study is the first experimental evidence that demonstrates the possibility of using this receptor as a target for cognitive impairment [3].

Note, theoretical studies were carried out for designing compounds with enhanced activity and selectivity upon I2-IR based on created 3D-QSAR model.

In this work, green chemistry to access an unprecedented scaffold and promising pharmacological results in the neurodegeneration field walked together. 
Acknowledgments: Financial support Projects SAF2016-77703-C2-1-R and 2017SGR106 is grateful acknowledged. A.B. thanks the Institute of Biomedicine of the Universitat de Barcelona (IBUB) and S.R.-A. thanks the Ministerio de Economia, Industria y Competitividad (FPI program) for their PhD grants.

\section{References}

1. Abas, S.; Estarellas, C.; Luque, F.J.; Escolano, C. Easy access to (2-imidazolin-4-yl) phosphonates by a microwave assisted multicomponent reaction. Tetrahedron 2015, 71, 2872-2581.

2. Abás, S.; Erdozain, A.M.; Keller, B.; Rodríguez-Arévalo, S.; Callado, L.F.; García-Sevilla, J.A.; Escolano, C. Neuroprotective Effects of a Structurally New Family of High Affinity Imidazoline I2 Receptor Ligands. ACS Chem. Neurosci. 2017, 8, 737-742.

3. Griñán-Ferré, C.; Vasilopoulou, F.; Abás, S.; Rodríguez-Arévalo, S.; Bagán, A.; Sureda, F.X.; Pérez, B.; Callado, L.F.; García-Sevilla, J.A.; García-Fuster, M.J.; et al. Behavioral and Cognitive Improvement Induced by Novel Imidazoline I2 Receptor Ligands in Female SAMP8 Mice. Neurotherapeutics 2018, 16, 416431, doi:10.1007/s13311-018-00681-5.

(C) 2019 by the authors. Licensee MDPI, Basel, Switzerland. This article is an open access article distributed under the terms and conditions of the Creative Commons Attribution (CC BY) license (http://creativecommons.org/licenses/by/4.0/). 